\title{
Statistical genetics and polygenic risk score for precision medicine
}

\author{
Takahiro Konuma ${ }^{1,2}$ and Yukinori Okada $a^{1,3,4^{*}}$
}

\begin{abstract}
The prediction of disease risks is an essential part of personalized medicine, which includes early disease detection, prevention, and intervention. The polygenic risk score (PRS) has become the standard for quantifying genetic liability in predicting disease risks. PRS utilizes single-nucleotide polymorphisms (SNPS) with genetic risks elucidated by genome-wide association studies (GWASs) and is calculated as weighted sum scores of these SNPs with genetic risks using their effect sizes from GWASs as their weights. The utilities of PRS have been explored in many common diseases, such as cancer, coronary artery disease, obesity, and diabetes, and in various non-disease traits, such as clinical biomarkers. These applications demonstrated that PRS could identify a high-risk subgroup of these diseases as a predictive biomarker and provide information on modifiable risk factors driving health outcomes. On the other hand, there are several limitations to implementing PRSs in clinical practice, such as biased sensitivity for the ethnic background of PRS calculation and geographical differences even in the same population groups. Also, it remains unclear which method is the most suitable for the prediction with high accuracy among numerous PRS methods developed so far. Although further improvements of its comprehensiveness and generalizability will be needed for its clinical implementation in the future, PRS will be a powerful tool for therapeutic interventions and lifestyle recommendations in a wide range of diseases. Thus, it may ultimately improve the health of an entire population in the future.
\end{abstract}

Keywords: Statistical genomics, Genome-wide association study, Polygenic risk score, Precision medicine

\section{Background}

Understanding human disease risk factors that contribute to disease onset is vital for the implementation of early disease detection, prevention, and intervention. The primary components of human disease risk factors are usually explained by the combination of genetic susceptibility, environmental exposures, and lifestyle factors [1]. Differences in these factors between individuals also yield differences in disease physiology among individuals. Precision medicine can be defined as tailored medical care primarily based on understanding these differences in disease physiology among individuals (Fig. 1a).

\footnotetext{
* Correspondence: yokada@sg.med.osaka-u.ac.jp

${ }^{1}$ Department of Statistical Genetics, Osaka University Graduate School of

Medicine, 2-2 Yamadaoka, Suita 565-0871, Japan

${ }^{3}$ Laboratory of Statistical Immunology, Immunology Frontier Research Center (WPI-IFReC), Osaka University, Suita 565-0871, Japan

Full list of author information is available at the end of the article
}

One of the important approaches for precision medicine is stratifying individual genetic susceptibility based on inherited DNA variation. This approach has been developed with progress in human genetics. Since the first complete human genome sequencing was finished in 2003, progress in human genetics has been accelerated by recent technological advances, such as genome sequencing technology for a large population and advances in statistical genetics methodology. All this progress in human genetics has been expected to give insight into the contribution of genetic factors for common human diseases and better prediction of disease risks. A genome-wide association study (GWAS), which uses single-nucleotide polymorphisms (SNPs) arrays, is one of the most effective methods for statistically assessing the genetic association of diseases. Not only have GWASs identified thousands of genomic loci associated with common human diseases [2], they have also

(c) The Author(s). 2021 Open Access This article is licensed under a Creative Commons Attribution 4.0 International License, which permits use, sharing, adaptation, distribution and reproduction in any medium or format, as long as you give appropriate credit to the original author(s) and the source, provide a link to the Creative Commons licence, and indicate if changes were made. The images or other third party material in this article are included in the article's Creative Commons licence, unless indicated otherwise in a credit line to the material. If material is not included in the article's Creative Commons licence and your intended use is not permitted by statutory regulation or exceeds the permitted use, you will need to obtain permission directly from the copyright holder. To view a copy of this licence, visit http://creativecommons.org/licenses/by/4.0/. 

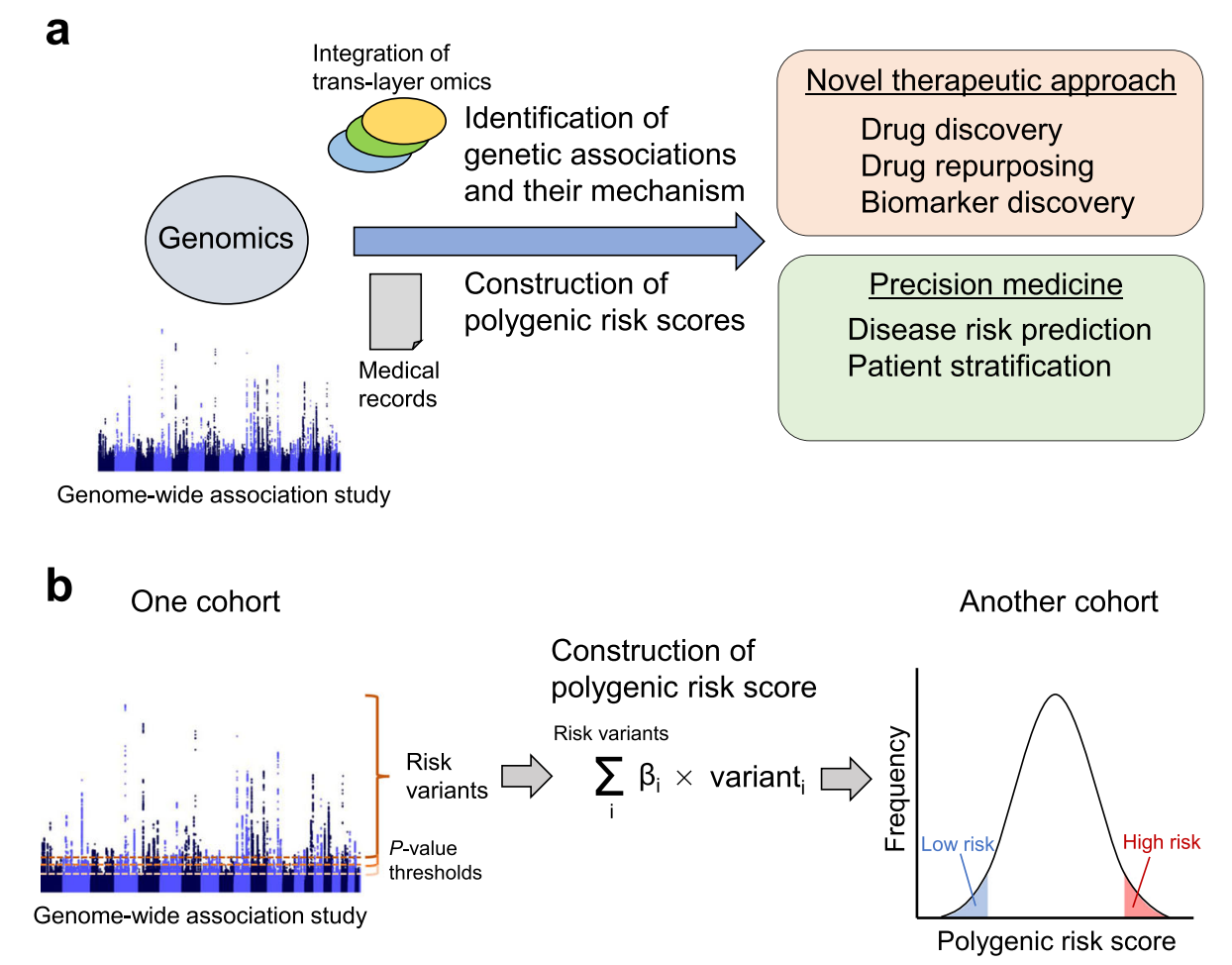

Another cohort

Fig. 1 Overview of clinical application of statistical genomics and polygenic risk score. a Clinical application of statistical genomics for complex human diseases. $\mathbf{b}$ Overview of polygenic risk score construction. As a usual practice, PRS is calculated as a weighted sum of several risk variants from a genome-wide association study in one cohort with multiple $p$-value thresholds. The effect sizes are typically estimated as $\beta$ (beta coefficients) or as odds ratios. After the PRS is calculated in one cohort, the distribution of individual PRS is assessed in another cohort

elucidated complex genetic architectures in most common human diseases. Because most SNPs identified by GWASs that are significantly associated with common human diseases often have a small effect size on these disease risks [3], using only SNPs that are significantly associated with these diseases in disease risk prediction is not reliable. A model underlying this concept is called a "polygenic model" [4], which explains disease susceptibility as a combination of several SNPs that have a small effect size on disease.

A statistical method based on a polygenic model, called the PRS, has been developed. PRS is calculated as a weighted sum of several risk alleles carried by an individual, where the risk alleles and their weights are defined by SNPs and their measured effects [5]. PRS has gained interest because it may be useful in predicting individual disease susceptibility. In this review, we summarize the recent advances, utilities, perspectives, and precision medicine application of PRS (Fig. 1).

\section{An overview of polygenic risk score}

The concept of polygenic risk was initially advocated and modeled in the early twentieth century [6]. Along with the accumulation of genomic loci associated with common human diseases and complex traits by the success of GWASs [2], it has been possible to quantify polygenic risk using risk alleles identified by GWASs in these diseases and traits. The quantifying polygenic risk methods have been developed in the last decade as tools to calculate the cumulative effect of many genetic loci for a certain trait into a quantitative metric [7], which is called PRS. As a usual practice, PRS is calculated as a weighted sum of several risk alleles carried by an individual. The risk alleles and their weights are defined by SNPs and their measured effect sizes (Fig. 1b). The effect sizes are typically estimated as the beta coefficients for quantitative traits or as odds ratios for categorical binary traits. PRS is typically calculated using a set of SNPs with different $p$-value thresholds (e.g., $1 \times 10^{-5}, 1 \times$ $\left.10^{-4}, \ldots, 0.05,0.1, \ldots, 0.5\right)$ for disease association, and then a series of PRSs is calculated for a disease or a trait. After the PRS has been calculated in one cohort, it is essential to assess its predictive performance in another external cohort, which is not used for the construction of PRS. This performance of constructed PRS is often evaluated by the area under the receiver operating characteristic curve, called area under the curve (AUC) of a PRS, which provides a quantitative measure for the discrimination ability of a PRS [8]. 
In the calculation and validation of PRS, some methodological concerns have been argued [9, 10]. For example, although the construction of PRS by inclusion of larger numbers of SNPs (including SNPs that do not meet genome-wide significance) can have more predictive accuracy, it is argued whether the inclusion of those SNPs with close to zero effects in PRSs makes sense [11]. In another example, linkage disequilibrium (LD), the correlations between nearby SNPs, which leads to over-representation of high LD regions in calculating PRS, potentially reduces the predictive performance of PRS [12]. To mitigate the effect of LD, LD pruning (randomly removing one SNP from a pair in high LD), LD clumping (pruning by LD, while referentially retaining more significantly associated SNPs), or more complex methods that explicitly account for LD [13] have been used.

\section{Applications of the polygenic risk score for disease prediction}

In the case of disease with age-dependent prevalence, such as lifestyle-related diseases, it is effective to identify the population with a high risk of disease onset in advance and implement a preventive intervention. One of the PRS utilities with high clinical values can be a predictive biomarker of disease risk. This utility of PRS has been explored in many common diseases, such as cancer, coronary artery disease, obesity, and diabetes [1416]. For example, in coronary artery disease, PRS, which was developed by a GWAS of coronary artery disease from a dataset (validation dataset) of UK Biobank participants and applied to another dataset (test dataset) of UK Biobank participants, demonstrated that participants in the top 0.5 percentile of PRS in the test dataset had a fivefold increase in the prevalence of coronary artery disease [16]. This result showed that PRS developed by a large-scale GWAS potentially enabled the accurate prediction of disease prevalence.

In another example, disease risk prediction of breast cancer, which had been estimated from two genes, BRCA1 and BRCA2 [17], was expanded by the application of PRS. PRS of breast cancer based on 303 genetic variants from a GWAS of breast cancer demonstrated that women in the top 1 percentile of PRS had a fourfold increased risk of developing estrogen receptor-positive breast cancer and a sixfold decreased risk for women in the lowest 1 percentile of PRS [18]. Although this PRS had a modest AUC of 0.63, this study showed that breast cancer PRS potentially captured sufficient information to identify a high-risk subgroup of women who could be offered preventive interventions.

Application of PRS for a non-disease trait was also reported. This analysis developed PRSs of trans-biobank (BioBank Japan, UK Biobank, and FinnGen; $n_{\text {total }}=675$,
898) analysis of the association of several clinical biomarkers and revealed the association between high systolic blood pressure PRS and a shorter lifespan in transethnic individuals and the association between obesity PRS and lifespan in Japanese and European individuals [19]. These results showed the potential application of PRS in improving population health by providing information on modifiable risk factors driving health outcomes.

From these examples, the utilities of PRS have been expected to be potential predictors of future risks of disease or health outcomes. Thus, they are expected for target treatment application, alteration of screening paradigms, and modification of non-genetic factors related to predicted high-risk phenotypes.

\section{Limitations and challenges for the application of polygenic risk score}

We focus on several limitations on the implementation of PRSs in clinical practice. First, PRS is highly sensitive to ethnic background. The variability of PRS among ethnic groups can be explained by the differences in allele frequency, LD, and effect sizes of variants among ethnic groups [20]. Therefore, the performance of PRS drops if PRS developed from one ethnic group is applied to another ethnic group [21]. To overcome these ethnic group-specific biases, several methods have been proposed. For example, the ancestry deconvolution PRS method with consideration for an admixture of ancestryspecific partial sequence in individual genome demonstrated improved susceptibility predictions of PRS for four traits (type 2 diabetes, breast cancer, height, and body mass index [BMI]) [22]. In addition to the further development of the PRS method, future GWASs would be needed to include subjects from diverse ethnic backgrounds to improve the generalizability and utility of PRS for all populations because the majority of GWASs have been performed in European-Caucasian populations $[23,24]$. In order to enlarge the benefit of PRS in non-European-Caucasian populations, whose amount of genomic data is limited compared with EuropeanCaucasian populations, it is important that vastly increasing diversity of participants is included and analyzed in genetic studies, and open data-sharing standards of these results are needed for improving the accuracy of PRS in these populations [24].

Second, the distribution of PRS even in the population group was reported to show biases according to geographical differences. For example, geographical differences in PRSs of coronary artery disease, rheumatoid arthritis, schizophrenia, waist-hip ratio, BMI, and height were detected in Finland [25]. Whether the cause of these biased distributions was the geographical difference in disease prevalence or the difference in the 
genetic background by population stratification remains unclear. In another example, considerable differences of PRSs between the non-mainlanders and mainlanders in Japan were reported [26]. In this report, PRS of BMI showed that the smaller BMI PRS was observed in nonmainlanders, although the greater BMI was observed in non-mainlanders. This difference is assumed to be a result of sudden changes in environmental factors which affected non-mainlanders' BMI in the non-mainland that preceded the reflection of genomic structure in response to these environmental changes. From these examples, the PRS could be susceptible to population structure's geographical distributions, even within a relatively homogeneous population.

Third, given the numerous available models of PRS, it remains unclear which method is the most suitable for predicting the risks of diseases or traits. It was reported that when categorizing the existing 15 PRS methods into three groups, which consisted of (1) simple methods that selected variants below a $p$-value limit and within a LD range, (2) complex methods that selected variants by attempting to approximate the results of a mixed-model approach, and (3) ensemble methods created by taking an average of the top five PRSs weighted by their coefficients in a cross-validated logistic regression, it was shown that the simple methods generated slightly more accurate PRSs than did the complex methods [27]. Further insight into the characterization of PRS models will be needed to evaluate and compare these predictive performances. Comparable performance metrics of these PRS models would need to be systematically evaluated. For example, the PGS Catalog [28], an open resource for PRSs that has been reported recently, enables PRS analysis in a standardized format along with consistent metadata and direct comparison between scores.

Fourth, the prospect of clinical use of PRS is associated with a wide variety of ELSI (ethical, legal, and social implications) concerns, which have been also discussed in the context of monogenic genetic results and is also present in the polygenic context [29]. One of the ELSI concerns about PRS is the relevance of findings of PRS to family members. Genetic variation is shared in families and the PRS of first-degree family members are correlated [30], but this information is not as clear as in the monogenic genetic results. Guidelines developed by professional societies would be needed for both patients and providers for prompt warning about the polygenic risk to family members. Other examples of the ELSI concerns about PRS are risk of psychosocial harms, false reassurance, and overdiagnosis and overtreatment, which are typically considered in the monogenic genetic results. Further research for whether the harms of false reassurance, overtreatment and overdiagnosis materialize would be needed.

\section{Conclusions}

In this review, we focused on recent advances, utilities, and perspectives of PRS. The predictive accuracy of PRS will continue to be improved by more extensive and diverse cohorts to construct PRS models and improve methods for PRS derivation and application. Although further improvements of its comprehensiveness and generalizability would be needed for its clinical implementation in the future, the potential clinical impacts and benefits of the PRS have been proposed and discussed. For example, PRS-informed clinical intervention, PRS-informed disease screening, and PRS-informed life planning were proposed as the potential clinical benefits [5]. Also, individual PRS measurement only needs its genome sequence, which could be taken once at a relatively low cost, and PRS will be potentially applied for various diseases and traits. PRS will guide therapeutic interventions and lifestyle recommendations in several diseases. Thus, it might ultimately improve the health of an entire population in the future.

\section{Abbreviations \\ PRS: Polygenic risk score; SNP: Single nucleotide polymorphism; GWAS: Genome-wide association study; AUC: Area under the curve; ER: Estrogen receptor; BMI: Body mass index}

\section{Acknowledgements}

Not applicable.

\section{Authors' contributions}

T.K. wrote the manuscripts. Y.O. supervised the review. The authors read and approved the final manuscript.

\section{Funding}

This study was supported by the Japan Society for the Promotion of Science (JSPS) KAKENHI (19H01021 and 20 K21834) and AMED (JP20km0405211, JP20ek0109413, JP20ek0410075, JP20gm4010006, and 20 km0405217), Takeda Science Foundation, and Bioinformatics Initiative of Osaka University Graduate School of Medicine, Osaka University. T.K. is an employee of Japan Tobacco Inc.

Availability of data and materials

Not applicable.

\section{Declarations}

Ethics approval and consent to participate Not applicable.

Consent for publication

Not applicable.

\section{Competing interests}

The authors declare that they have no competing interests.

\section{Author details}

${ }^{1}$ Department of Statistical Genetics, Osaka University Graduate School of Medicine, 2-2 Yamadaoka, Suita 565-0871, Japan. ${ }^{2}$ Central Pharmaceutical Research Institute, Japan Tobacco Inc., Takatsuki 569-1125, Japan. ${ }^{3}$ Laboratory of Statistical Immunology, Immunology Frontier Research Center (WPI-IFReC), Osaka University, Suita 565-0871, Japan. ${ }^{4}$ Integrated Frontier Research for Medical Science Division, Institute for Open and Transdisciplinary Research Initiatives, Osaka University, Suita 565-0871, Japan. 
Received: 28 April 2021 Accepted: 9 June 2021

Published online: 17 June 2021

\section{References}

1. Torkamani A, Wineinger NE, Topol EJ. The personal and clinical utility of polygenic risk scores. Nat Rev Genet. 2018;19:1-10.

2. Visscher PM, Wray NR, Zhang Q, Sklar P, McCarthy Ml, Brown MA, et al. 10 years of GWAS discovery: biology, function, and translation. Am J Hum Genet. 2017;101(1):5-22. https://doi.org/10.1016/j.ajhg.2017.06.005.

3. Manolio TA, Collins FS, Cox NJ, Goldstein DB, Hindorff LA, Hunter DJ, et al. Finding the missing heritability of complex diseases. Nature. 2009:461(7265): 747-53. https://doi.org/10.1038/nature08494

4. Golan D, Lander ES, Rosset S. Measuring missing heritability: inferring the contribution of common variants. Proc Natl Acad Sci U S A. 2014;111(49): E5272-81. https://doi.org/10.1073/pnas.1419064111.

5. Torkamani A, Wineinger NE, Topol EJ. The personal and clinical utility of polygenic risk scores. Nat Rev Genet. 2018;19(9):581-90. https://doi.org/10.1 038/s41576-018-0018-x.

6. Fisher RA. The correlation between relatives on the supposition of Mendelian inheritance. Trans R Soc Edinb. 1919;52(2):399-433. https://doi. org/10.1017/S0080456800012163.

7. Wray NR, Goddard ME, Visscher PM. Prediction of individual genetic risk to disease from genome-wide association studies. Genome Res. 2007;17(10): 1520-8. https://doi.org/10.1101/gr.6665407.

8. Hanley JA, McNeil BJ. The meaning and use of the area under a receiver operating characteristic (Roc) curve. Radiology. 1982;143(1):29-36. https:// doi.org/10.1148/radiology.143.1.7063747.

9. Choi SW, Mak TSH, O'Reilly PF. Tutorial: a guide to performing polygenic risk score analyses. Nat Protoc. 2020;15(9):2759-72. https://doi.org/10.1038/s41 596-020-0353-1.

10. Chatterjee N, Shi J, García-Closas M. Developing and evaluating polygenic risk prediction models for stratified disease prevention. Nat Rev Genet. 2016; 17(7):392-406. https://doi.org/10.1038/nrg.2016.27.

11. Janssens ACJW, Joyner MJ. Polygenic risk scores that predict common diseases using millions of single nucleotide polymorphisms: is more, better? Clin Chem. 2019;65(5):609-11. https://doi.org/10.1373/clinchem.2018.296103.

12. Wu J, Pfeiffer RM, Gail MH. Strategies for developing prediction models from genome-wide association studies. Genet Epidemiol. 2013;37(8):768-77. https://doi.org/10.1002/gepi.21762.

13. Vilhjálmsson BJ, Yang J, Finucane HK, Gusev A, Lindström S, Ripke S, et al. Modeling linkage disequilibrium increases accuracy of polygenic risk scores. Am J Hum Genet. 2015;97(4):576-92. https://doi.org/10.1016/j.ajhg.2015.09. 001.

14. Fritsche LG, Beesley L, VandeHaar P, Peng RB, Salvatore M, Zawistowski M, et al. Exploring various polygenic risk scores for skin cancer in the phenomes of the Michigan genomics initiative and the UK Biobank with a visual catalog: PRSWeb. PLoS Genet. 2019;15(6):e1008202. https://doi.org/1 0.1371/journal.pgen.1008202.

15. Khera AV, Chaffin M, Wade KH, Zahid S, Brancale J, Xia R, et al. Polygenic prediction of weight and obesity trajectories from birth to adulthood. Cell. 2019;177:587-96.e9.

16. Khera AV, Chaffin M, Aragam KG, Haas ME, Roselli C, Choi SH, et al. Genome-wide polygenic scores for common diseases identify individuals with risk equivalent to monogenic mutations. Nat Genet. 2018;50(9):121924. https://doi.org/10.1038/s41588-018-0183-z

17. Antoniou AC, Cunningham AP, Peto J, Evans DG, Lalloo F, Narod SA, et al. The BOADICEA model of genetic susceptibility to breast and ovarian cancers: updates and extensions. Br J Cancer. 2008;98(8):1457-66. https:// doi.org/10.1038/sj.bjc.6604305.

18. Mavaddat N, Michailidou K, Dennis J, Lush M, Fachal L, Lee A, et al. Polygenic risk scores for prediction of breast cancer and breast cancer subtypes. Am J Hum Genet. 2019;104(1):21-34. https://doi.org/10.1016/j.a jhg.2018.11.002.

19. Sakaue S, Kanai M, Karjalainen J, Akiyama M, Kurki M, Matoba N, et al. Transbiobank analysis with 676,000 individuals elucidates the association of polygenic risk scores of complex traits with human lifespan. Nat Med. 2020; 26(4):542-8. https://doi.org/10.1038/s41591-020-0785-8.

20. Márquez-Luna C, Loh P. South Asian Type 2 Diabetes (SAT2D) Consortium, SIGMA Type 2 Diabetes Consortium, Price AL. Multiethnic polygenic risk scores improve risk prediction in diverse populations. Genet Epidemiol. 2017;41(8):811-23. https://doi.org/10.1002/gepi.22083.
21. Duncan L, Shen H, Gelaye B, Meijsen J, Ressler K, Feldman M, et al. Analysis of polygenic risk score usage and performance in diverse human populations. Nat Commun. 2019;10(1):3328. https://doi.org/10.1038/s41467019-11112-0.

22. Marnetto D, Pärna K, Läll K, Molinaro L, Montinaro F, Haller T, et al. Ancestry deconvolution and partial polygenic score can improve susceptibility predictions in recently admixed individuals. Nat Commun. 2020;11(1):1628. https://doi.org/10.1038/s41467-020-15464-w.

23. Mills MC, Rahal C. A scientometric review of genome-wide association studies. Commun Biol. 2019;2(1):9. https://doi.org/10.1038/s42003-018-0261-X.

24. Martin AR, Kanai M, Kamatani Y, Okada Y, Neale BM, Daly MJ. Clinical use of current polygenic risk scores may exacerbate health disparities. Nat Genet. 2019;51(4):584-91. https://doi.org/10.1038/s41588-019-0379-x.

25. Kerminen S, Martin AR, Koskela J, Ruotsalainen SE, Havulinna AS, Surakka I, et al. Geographic variation and bias in the polygenic scores of complex diseases and traits in Finland. Am J Hum Genet. 2019;104(6):1169-81. https://doi.org/10.1016/j.ajhg.2019.05.001.

26. Sakaue S, Hirata J, Kanai M, Suzuki K, Akiyama M, Too CL, et al. Dimensionality reduction reveals fine-scale structure in the Japanese population with consequences for polygenic risk prediction. Nat Commun. 2020;11(1):1569. https://doi.org/10.1038/s41467-020-15194-z.

27. Kulm S, Mezey J, Elemento O. Benchmarking the accuracy of polygenic risk scores and their generative methods. medRxiv. 2020. https://doi.org/10.11 01/2019.12.11.12345678

28. Lambert SA, Gil L, Jupp S, Ritchie S, Xu Y, Buniello A, et al. The Polygenic Score Catalog: an open database for reproducibility and systematic evaluation. Nat Genet. 2021;53(4):420-5. https://doi.org/10.1038/s41588-02100783-5.

29. Lewis ACF, Green RC. Polygenic risk scores in the clinic: new perspectives needed on familiar ethical issues. Genome Med. 2021;13(1):14. https://doi. org/10.1186/s13073-021-00829-7.

30. Karavani E, Zuk O, Zeevi D, Barzilai N, Stefanis NC, Hatzimanolis A, et al. Screening human embryos for polygenic traits has limited utility. Cell. 2019; 179:1424-1435.e8

\section{Publisher's Note}

Springer Nature remains neutral with regard to jurisdictional claims in published maps and institutional affiliations.
Ready to submit your research? Choose BMC and benefit from:

- fast, convenient online submission

- thorough peer review by experienced researchers in your field

- rapid publication on acceptance

- support for research data, including large and complex data types

- gold Open Access which fosters wider collaboration and increased citations

- maximum visibility for your research: over $100 \mathrm{M}$ website views per year

At BMC, research is always in progress.

Learn more biomedcentral.com/submissions 